\title{
Crecimiento Económico como determinante de los fujos de efectivo para las empresas del sector industrial de Caldas - Colombia, durante el periodo $1995-2013^{*}$
}

Economic growth as a determinant of cash flows for companies

in the industrial sector of Caldas - Colombia, 1995 - 2013

O crescimento econômico como determinante dos fluxos de caixa para as empresas do sector industrial de Caldas - Colômbia, 1995 2013

Recibido el 19 de julio de 2016. Aceptado el 11 de noviembre de 2016

Gabriel Eduardo Escobar Arias**

Colombia

, Para citar este artículo:

Escobar Arias, Gabriel Eduardo (diciembre, 2016). Crecimiento Económico como determinante de los flujos futuros de efectivo

para las empresas del sector industrial de Caldas - Colombia, durante el periodo 1995 - 2013

Ánfora, 23(41), 179-201. Universidad Autónoma de Manizales. ISSN 0121-6538.

\section{Resumen}

Objetivo: determinar la relación entre el crecimiento económico y los flujos de efectivo de las empresas del sector industrial en Caldas. Metodología: se partió de los estados financieros de 47 empresas industriales de Caldas que reportaron sus estados financieros a la Superintendencia de Sociedades durante 1995; este número de empresas constituyó la población inicial del estudio. Sin embargo, finalmente el análisis se aplicó a 12 empresas que reportaron de forma continua

\footnotetext{
* El presente artículo se deriva de la investigación titulada "Crecimiento económico y flujos de efectivo para el sector industrial en el departamento de Caldas durante el periodo 1995 - 2013. Las instituciones que auspiciaron el estudio fueron la Universidad Autónoma de Manizales y la Universidad Nacional de Colombia sede Manizales. El proceso investigativo inició en el mes de abril de 2014 y fue finalizado en mayo de 2016.

** Doctor en ingeniería industria y organizaciones. Economista. Profesor asociado de la Universidad Autónoma de Manizales. Profesor asociado de la Universidad Nacional de Colombia sede Manizales. Correo electrónico: gabrieledo@autonoma.edu.co
} 
sus estados financieros a la Superintendencia de Sociedades de Colombia de 1995 a 2013. A estas empresas se les calculó los flujos de efectivo de operación y financiación con los cuales se estableció la relación estadística con el crecimiento de la economía regional durante el mismo periodo; estos datos se obtuvieron del Departamento Nacional de Estadística (DANE) de Colombia. Resultados: la relación estadística entre los flujos de efectivo de operación y el crecimiento de la economía regional ascendió al 0,43 , resultado insatisfactorio para realizar predicciones, pero al sumarle a los flujos de efectivo de operación la financiación, la relación estadística aumentó a 0,77, dato más preciso para hacer la predicción de los futuros flujos de efectivo. Conclusiones: se concluye que por relación directa y alta entre los flujos de efectivo de las empresas del sector industrial del Departamento de Caldas y el crecimiento económico regional, se pueden realizar predicciones de los futuros flujos de efectivo para las empresas del sector, de tal forma que gremios y empresarios puedan realizar una mejor planeación financiera en sus organizaciones.

Palabras clave: Flujos de efectivo; Crecimiento económico.

\section{Abstract}

Objective: to determine the relationship between economic growth and cash flows of companies in the industrial sector of Caldas. Methodology: the financial statements of 47 industrial companies in Caldas, which reported their financial statements to the Superintendency of Companies during 1995, were taken into account; this number of companies constituted the initial study population. However, finally the analysis was applied to the 12 companies that continuously reported their financial statements to the Superintendency of Societies of Colombia from 1995 to 2013. The operating and financing cash flows estimated for these companies were calculated, which allowed the establishment of the statistical relationship with the growth of the regional economy during the same period. This data was obtained from the National Administrative Statistics Department of Colombia (DANE). Results: the statistical relationship between the operating cash flows and the growth of the regional economy amounted to 0.43 , which is an unsatisfactory result to make predictions. However, when adding financing cash flows to the operating ones, the statistical ratio increased to 0.77. This data allows for more accurate predictions of future cash flows. Conclusions: it was concluded that direct and high relationships between the cash flows of the companies in the industrial sector of the Department of Caldas and the regional economic growth enables predictions to be made of future cash flows for companies in the sector, so that unions and employers can make better financial planning in their organizations.

Keywords: Cash flows; Economic growth. 


\section{Resumo}

Objetivo: determinar a relação entre o crescimento económico e os fluxos de caixa das empresas do setor industrial em Caldas. Metodologia: partiu-se dos estados financeiros de 47 empresas industriais de Caldas que reportaram seus estados financeiros à Superintendência de sociedades durante 1995; este número de empresas foi a população inicial do estudo. No entanto, finalmente, a análise foi aplicada a 12 empresas que reportaram de forma continua seus estados financeiros à Superintendência de Sociedades da Colômbia de 1995 a 2013. Os fluxos de caixa de operação e financiamento destas empresas foram calculados e com esta informação estabeleceu-se a relação estatística com o crescimento da economia regional durante o mesmo período; Estes dados foram obtidos do Departamento Nacional de Estatística (DANE) da Colômbia. Resultados: a relação estatística entre os fluxos de caixa operacional e o crescimento da economia regional ascendeu a 0,43, resultado insatisfatório para fazer previsões, mas no momento de adicionar aos fluxos de caixa de operação o financiamento, a relação estatística aumentou para 0,77, cifra mais precisa para fazer a predição dos fluxos de caixa futuros. Conclusões: concluiu-se que por relação direta e alta entre os fluxos de caixa das empresas do setor industrial do Departamento de Caldas e o crescimento económico regional, podem-se fazer futuras previsões de fluxos de caixa para as empresas do setor, de forma que grêmios e empregadores podam fazer um planejamento financeiro melhor em suas organizações.

Palavras-chave: Fluxos de caixa; Crescimento económico. 


\section{Introducción}

En procesos investigativos anteriores se analizaron los flujos de efectivo de algunas empresas del departamento de Caldas, Risaralda y Quindío (Colombia) para el sector comercial, de servicios y agropecuario, entre 2002 y $2010^{1}$. Tal estudio revisó sus estructuras de operación, financiación e inversión y determinó su relación con algunas variables macroeconómicas como el nivel de empleo, la inflación, la tasa representativa del mercado, el producto interno bruto y el índice de la bolsa de valores de Colombia, durante el mismo período. Algunos hallazgos demostraron la correlación positiva entre las variables analizadas como lo fueron el producto interno bruto, el nivel de empleo, la tasa representativa del mercado, entre otras, con los flujos de efectivo.

De acuerdo con lo anterior, se evidencia un vacío teórico de la relación entre los resultados de los flujos de efectivo y algunas variables macroeconómicas. Por ello, se indagó en profundidad sobre la relación que pueden presentar variables como el crecimiento económico y los flujos de efectivo y su incidencia en éstos, de forma que se pueda realizar su predicción para empresas del sector industrial en Caldas con una muestra de 12 empresas.

Ou y Penman (1989), Sloan (1996), Luo (2008), Lorek (2014), entre otros, presentaron resultados sobre la predicción de los flujos de efectivo para períodos siguientes, a partir de variables como los flujos de efectivos históricos, los estados financieros principales, conformados por el balance general y el estado de resultados o situación económica, el capital de trabajo, los ingresos, los costos y otros informes financieros, redactados bajo normas internacionales de información financiera (NIIF). Una de sus conclusiones es que para realizar una predicción más exacta de los flujos de efectivo, se debe hacer uso de variables adicionales aunque no señalan cuales. Sin embargo, ninguno de ellos, en la búsqueda realizada en las bases de datos consultadas, trabajó la predicción a través de la relación que pueda haber entre el crecimiento de la economía y los flujos de efectivo históricos.

En ese sentido se observa un vacío teórico entre la relación que puedan tener estas dos variables y la manera de conjugarlas para establecer una predicción de los flujos de efectivo a partir de estos resultados. De acuerdo con lo anterior,

1 Investigación finalizada en el año 2011 titulada relaciones entre los flujos de efectivo y variables macroeconómicas para los sectores comercial, servicios y agropecuario en los departamentos de Caldas, Risaralda y Quindío, Resultados publicados en la revista Reto del Servicio Nacional de Aprendizaje (SENA). Vol. 3 - Número 3 Junio de 2015. 
surgió la siguiente pregunta de investigación ¿Cuál es la relación entre el crecimiento económico y los flujos de efectivo en las empresas del sector industrial para Caldas entre los años 1995 y 2013 ?

Así, los objetivos del presente trabajo son, en primer lugar, establecer la relación teórica y práctica entre el crecimiento económico y los flujos de efectivo; segundo, establecer la correlación entre el crecimiento de la economía y los flujos de efectivo durante los años 1995 a 2013 de las empresas del sector industrial en Caldas. Y, finalmente, como consecuencia del estudio, plantear un sistema que permita proyectar los flujos de efectivo a partir de la variación que presenta el crecimiento de la economía. Es decir, medir el porcentaje de variación que presentarán los flujos de efectivo ante la variación del PIB de la economía regional en la cual se basa el estudio. Esta observación se fundamenta en que la variable independiente será el crecimiento económico y la variable dependiente serán los flujos de efectivo.

Con los resultados, los empresarios podrán tener información adicional para realizar sus pronósticos respecto a los flujos de efectivo, pues cuentan con información interna y externa y de esta forma podrán realizar una planeación financiera más adecuada para prever su capital de trabajo, las inversiones futuras, el pago de dividendos y de obligaciones financieras, entre otras actividades relacionadas con el desembolso o la adquisición de recursos monetarios o liquidez.

Se presenta, además, un análisis en el cual se estableció la relación que pueden tener algunos hechos económicos con los flujos de efectivo para las regiones y durante el mismo tiempo, de tal forma que se pueda demostrar que las correlaciones entre las dos variables se pueden explicar de forma cuantitativa y cualitativa.

Finalmente, se espera que los empresarios del sector, los gremios y la academia puedan contar con una herramienta adicional para tomar decisiones que apunten al crecimiento de sus organizaciones, pues al contar con mayor información, pueden obtener resultados más certeros respecto a los flujos de efectivo.

\section{Metodología}

Uno de los objetivos de la investigación fue evidenciar la relación entre el crecimiento económico regional y los flujos de efectivo; por tanto, la investigación que se presenta en este informe es de tipo cuantitativo y correlacional. Se 
estableció en esta correlación el efecto de la variable independiente, que es el crecimiento económico, en la variable dependiente representada por los flujos de efectivo. Además, la investigación fue de carácter longitudinal, puesto que evaluó varios periodos continuos en cada una de las variables: el crecimiento económico y los flujos de efectivo. El periodo analizado es de 1995 a 2013.

Para la recolección de la información se utilizó la base de datos (SIREM) que es operada y suministrada de forma gratuita por la superintendencia de sociedades. Esta base de datos se encuentra en la página Web de la entidad (www. supersociedades.gov.co); la ruta establecida para llegar a dicha base de datos es en el link asuntos económicos y contables.

Para determinar el Producto Interno Bruto proyectado, se tomó la información suministrada por el Banco Mundial, entidad que tiene proyecciones de crecimiento PIB estimadas hasta el año 2017.

Para determinar la población se estableció el número de empresas del sector industrial del Departamento de Caldas (Colombia), que reportaron estados financieros a la Superintendencia de Sociedades durante 1995, de las cuales 47 empresas cumplieron con este requisito. Sin embargo, para determinar la muestra, se tomaron las empresas que reportaron estados financieros a la Superintendencia de Sociedades entre 1995 y 2013 de forma continua. Se decidió de esta manera, ya que en los periodos en los cuales no hay reporte, para la elaboración del aplicativo estadístico se tendría un dato cero, que afectaría los resultados de la correlación estadística. Para Caldas, objeto del presente estudio, se obtuvo el siguiente número de empresas:

Tabla 1. Empresas del sector industrial que reportaron estados financieros para Caldas, de forma continua a la Superintendencia de Sociedades durante el periodo 1995 - 2013.

\begin{tabular}{|c|c|}
\hline Departamento / región & Número de empresas \\
\hline Caldas & 12 \\
\hline
\end{tabular}

Fuente: elaboración propia con datos obtenidos de la Superintendencia de Sociedades.

Para el logro de los objetivos, se operó de la siguiente manera:

- Se contó con los históricos del Producto Interno Bruto (PIB) desde 1995 hasta 2013. 
- Se calcularon los flujos de efectivo de las empresas estudiadas a partir de los estados financieros Balance General y Estado de Resultados, datos suministrados por la Superintendencia de Sociedades entra 1995 y 2013.

- Se obtuvo la sumatoria de los flujos de efectivo de las 12 empresas del sector industrial de las regiones a las que se les aplicó el modelo estadístico.

- A través del software estadístico Statistical Package for the Social Sciences (SPSS) se estableció el grado de asociación lineal entre el PIB y los flujos de efectivo (relación), dato que entregó información importante para determinar el modelo econométrico que se utilizó para establecer los flujos de efectivo futuros.

- Se realizó una descripción de los hechos económicos ocurridos para cada región entre 1995 y 2013 con el objeto de determinar la relación de estos con los resultados de los flujos de efectivo.

- Para la estimación de los flujos de efectivo futuros para el período, de acuerdo con la estimación del PIB proyectado, se realizó mediante un modelo de regresión lineal de los flujos de efectivo en función del PIB de la siguiente forma

$$
\text { F.E.E. }=\text { Constante }+(\text { C } \times \text { C.E. })+\text { Error }^{2}
$$

De donde:

F.E.E. = Flujo de efectivo esperado.

$\mathrm{C}=$ Coeficiente del PIB.

C.E. = Crecimiento económico esperado.

Para la comprobación y la validez de la regresión de los datos y del modelo planteado, se realizaron pruebas estadísticas como La T de Student, que consiste en comprobar si "dos grupos difieren entre sí de manera significativa respecto a sus medias" Sampieri, Collado y Lucio (2006). Y Para determinar la validez del modelo, el nivel de significancia de la prueba debe ser menor a 0,05 (Creswell, 2005).

Otra prueba que se debe aplicar al modelo de regresión es la de Fisher (1925) que examina la igualdad entre las varianzas poblacionales de dos variables con distribución normal. Para la aceptación de la prueba bajo este parámetro, su nivel de significancia debe ser menor a 0,05 (Fisher, 1925). La tercera prueba es el coeficiente de determinación, que muestra el valor que puede explicar el efecto 
de la variable independiente en la variable dependiente. En la medida que ésta se acerque más a 1, significa que hay mayor grado de correlación y, por eso, los datos son más homogéneos para realizar modelos de predicción (Creswell, 2005).

Por su parte, Sampieri, Collado y Lucio (2006) utilizan una escala para determinar el grado de correlación, indicando que cuando el coeficiente de correlación está entre 0,25 y 0,50 es una correlación media, cuando está entre 0,50 y 0,75 es una correlación positiva y mayor a estos intervalos se considera que la correlación es muy alta.

La siguiente prueba estadística para determinar el modelo de regresión lineal es la normalidad, que muestra si los datos de las variables analizadas tienen una distribución normal, en la medida en que su nivel de significancia sea menor a 0,05 indica que los datos son homogéneos; es decir, que están dentro de la distribución normal. Por tanto, los datos son confiables para construir modelos de predicción (León y Montero, 2003).

La última prueba que se utiliza para comprobar la validez del modelo es la Homocedasticidad, que consiste en determinar la igualdad que presentan las varianzas de las variables analizadas. Para determinar que la prueba es válida, su resultado debe ser superior al 5\% de acuerdo con la prueba de Levene (Aranaz, 2002). Se utilizó el modelo de regresión lineal porque determina el efecto de una variable sobre otra (Sampieri, Collado y Lucio, 2006); de modo que, para el caso del presente trabajo, permite determinar el efecto del crecimiento económico como variable independiente, en los flujos de efectivo como variable dependiente.

\section{Resultados}

En los resultados que se exponen a continuación se presenta inicialmente la relación teórica existente entre el crecimiento de la economía y los flujos de efectivo y enseguida se presentan los resultados de la correlación entre las variables; el crecimiento económico la variable independiente y los flujos de efectivo la variable dependiente. Finalmente, se muestran los resultados de la predicción de los futuros flujos de efectivo a partir de la variación del crecimiento económico.

Se encontró que la principal variable que une los flujos de efectivo y el crecimiento económico es la liquidez que le pueda proporcionar el sistema financiero, como los flujos de efectivo, a las empresas, para que éstas puedan realizar la inversión necesaria y aporten al crecimiento de la economía. Esto se sustenta 
en las relaciones entre los agentes económicos, como lo señalan Aceves y Martínez (2013), quienes demostraron que, en un sistema financiero eficiente que le otorga recursos a las empresas a través del crédito, estos recursos son invertidos de manera eficiente para el crecimiento de la economía; tales recursos (liquidez) provienen de los ahorros de las empresas y las familias. Como se observa en el siguiente gráfico:

\section{Grafico 1. Relaciones entre los agentes económicos.}

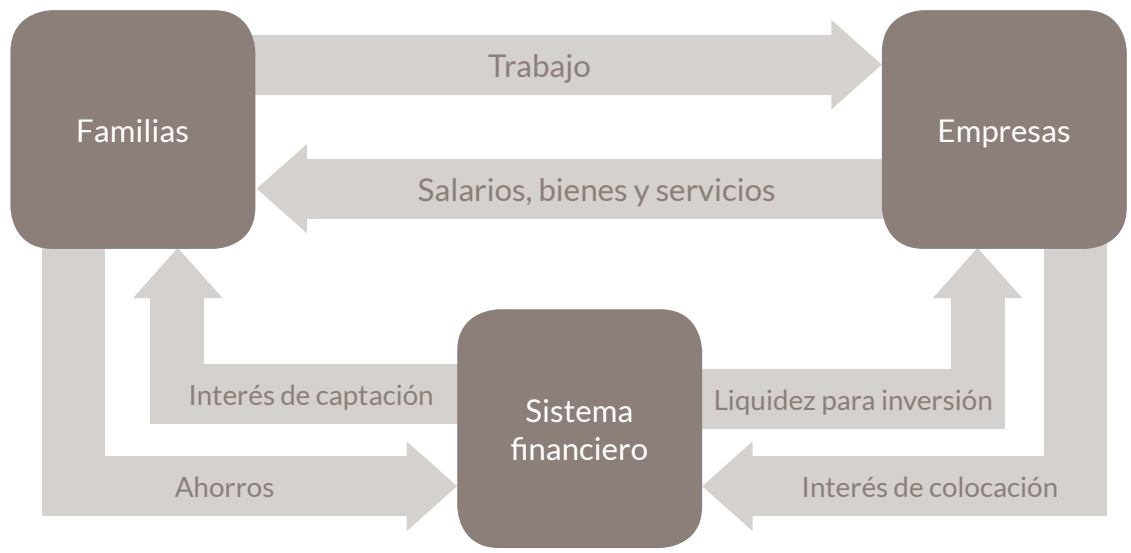

Fuente: elaboración propia.

Según el gráfico, las familias proveen a las empresas el trabajo para que éstas puedan producir bienes y servicios que luego serán ofrecidos a las familias para satisfacer sus necesidades. Por su parte, las familias reciben un salario por la actividad laboral en las empresas. De este salario (ingreso) las familias destinan una parte al consumo y otra al ahorro. Este ahorro es captado por el sistema financiero que se encarga de suministrar los recursos a las empresas para que realicen sus inversiones. Las familias, por llevar sus ahorros al sistema financiero, reciben una compensación conocida como tasa de interés de captación y las empresas reconocen al sistema financiero una tasa por usar los dineros prestables y que se le conoce como tasa de interés de colocación. La diferencia entre la tasa de interés de captación y de colocación se le llama tasa de intermediación financiera.

También muestra el gráfico que el sistema financiero se encarga de entregar la liquidez a las empresas para que realicen las inversiones necesarias y produzcan más y ofrezcan más bienes y servicios a la economía. En la medida que 
se produzca más, las familias recibirán mayor ingreso y podrán consumir más y ahorrar más. En la medida que esto se dé, como lo indicó Solow (1956) en su modelo de crecimiento económico, habrá mayor inversión en capital y por tanto mayor crecimiento económico.

Las empresas también pueden disponer de mayores recursos (flujos de efectivo) mediante la utilidad, dado que, en la medida en que produzcan más y vendan más, dispondrán de una mayor liquidez que podrá ser destinada para el incremento del capital.

El sistema financiero se encarga de proveer parte de la liquidez necesaria que demandan las empresas para realizar sus inversiones; se dice que esta provisión es parcial porque el total de la liquidez proviene de la suma de los créditos y los excedentes de liquidez que las empresas obtienen por su ejercicio comercial. Para que el sistema financiero provea esta liquidez, ofrece varios instrumentos de financiación para que los empresarios, de acuerdo con sus necesidades y requerimientos, puedan escoger las opciones que más se ajusten a sus necesidades y poder realizar las inversiones necesarias (Levine, 1997).

Con el tiempo, para proveer estos recursos, el sistema financiero se ha especializado en la formación de diferentes tipos de entidades que colocarán los ahorros en recursos para que las empresas puedan realizar sus inversiones. Entre ellas, hay algunas como los bancos, las corporaciones financieras, la bolsa de valores, la banca de segundo piso y las fiduciarias.

Terceño y Guercio (2011) demuestran que el desarrollo del sistema financiero interviene en forma directa en el crecimiento económico, es decir, hay una correlación entre el sistema financiero y el crecimiento. El estudio realizado por estos autores fue aplicado en países latinoamericanos, entre ellos Colombia.

En un estudio realizado entre 1990 y 2007, Terceño y Guercio (2011) realizaron una comparación entre el desarrollo del sistema financiero y su incidencia en el crecimiento económico en países como Argentina, Brasil, Chile, Colombia, México, Perú y Venezuela. Encontraron que la correlación entre el desarrollo del sistema financiero y el crecimiento económico es "alta e indiscutible” y la correlación más alta entre estas dos variables se dio en países como Brasil, Colombia, México y Perú.

Para determinar la correlación, los autores utilizaron como variable principal la capitalización del mercado de bonos. Este instrumento de financiación, provee 
liquidez a las empresas para que estas puedan realizar sus inversiones. En este sentido, encontraron que en Colombia la correlación es de 0,90 frente a un valor de uno (1), que es una correlación casi perfecta. Esto indica que en Colombia la inyección de liquidez por el sistema financiero para aportar al crecimiento de la economía es alta a través del mercado de bonos. Esta conclusión se observa también a través de la evolución de operaciones que realiza diariamente la bolsa de valores de Colombia; según datos de la entidad, entre 2001 hasta la fecha (en 2001 fue creada la BVC en Colombia, producto de la fusión de las bolsas de Medellín, Bogotá y Cali) el mercado de bonos representa el 75\% promedio del total de transacciones diarias realizadas.

Otro autor que señala que en Colombia se da el crecimiento económico basado en el mercado financiero es Ruiz (2004), quien, mediante un análisis econométrico en el que utiliza como variables el mercado accionario y de bonos, encontró que hay una alta relación estadística entre la liquidez que proveen los instrumentos financieros y el crecimiento de la economía.

En Colombia, hay varios tipos de entidades financieras que cumplen su función de intermediación al otorgarle la liquidez necesaria a las empresas para que puedan llevar a cabo sus inversiones y apoyar al crecimiento de la economía; entre estas se tiene:

Tabla 2. Entidades que inyectan liquidez a las empresas y las familias en Colombia

\begin{tabular}{|c|c|c|}
\hline Entidad & Tipo de productos & Colocaciones (clientes) \\
\hline Bancos & $\begin{array}{l}\text { Cuenta de ahorros, cuentas co- } \\
\text { rrientes, banca seguros, crédito de } \\
\text { libre inversión, fomento, tesorería } \\
\text { recursos de largo plazo, recursos } \\
\text { de corto plazo, vehículo, vivienda, } \\
\text { capital de trabajo, pymes, leasing, } \\
\text { factoring, descuentos a plazos, } \\
\text { cartas de crédito, fiducias, entre } \\
\text { otros productos de colocación y } \\
\text { captación. }\end{array}$ & $\begin{array}{l}\text { Personas naturales. } \\
\text { Grandes empresas. } \\
\text { Medianas empresas. } \\
\text { Pequeñas empresas. }\end{array}$ \\
\hline
\end{tabular}




\begin{tabular}{|c|c|c|}
\hline Entidad & Tipo de productos & Colocaciones (clientes) \\
\hline $\begin{array}{l}\text { Corporaciones } \\
\text { financieras }\end{array}$ & $\begin{array}{l}\text { Captación de recursos a través de } \\
\text { Certificados de depósito a térmi- } \\
\text { no, colocación de recursos para } \\
\text { promover la industria Colombiana } \\
\text { a través de créditos de largo plazo. }\end{array}$ & $\begin{array}{l}\text { Industria (hoy en Colombia sola- } \\
\text { mente hay dos entidades de este } \\
\text { tipo pues en la crisis de finales } \\
\text { de los noventa y principios de la } \\
\text { década de dos mil, la mayoría de } \\
\text { estas entidades desaparecieron. } \\
\text { Otro motivo es la poca capacidad } \\
\text { de diversificar productos financie- } \\
\text { ros al realizar sus captaciones y } \\
\text { colocaciones). }\end{array}$ \\
\hline $\begin{array}{l}\text { Compañías de } \\
\text { financiamiento } \\
\text { comercial }\end{array}$ & $\begin{array}{l}\text { Captación de recursos a través de } \\
\text { Certificados de depósito a térmi- } \\
\text { no y colocación de recursos para } \\
\text { promover el comercio a través de } \\
\text { créditos de mediano y corto plazo. }\end{array}$ & $\begin{array}{l}\text { Comercializadoras. Entidades que } \\
\text { dejaron de existir en Colombia } \\
\text { por los mismos motivos que se } \\
\text { presentaron para las corporacio- } \\
\text { nes financieras. }\end{array}$ \\
\hline $\begin{array}{l}\text { Compañías } \\
\text { especializadas en } \\
\text { leasing }\end{array}$ & $\begin{array}{l}\text { Sistema de arrendamiento } \\
\text { financiero utilizado por muchas } \\
\text { empresas para recomponer sus } \\
\text { activos de capital. Hay varios tipos } \\
\text { de leasing: financiero, operativo, } \\
\text { inmobiliario, sindicado, de impor- } \\
\text { tación, internacional. }\end{array}$ & $\begin{array}{l}\text { Personas naturales. } \\
\text { Grandes, medianas y pequeñas } \\
\text { empresas. }\end{array}$ \\
\hline Fiduciarias & $\begin{array}{l}\text { Contrato de administración de } \\
\text { recursos monetarios, de activos } \\
\text { inmobiliarios y fideicomisos admi- } \\
\text { nistrativos. }\end{array}$ & $\begin{array}{l}\text { Personas naturales. } \\
\text { Personas jurídicas. } \\
\text { (No es un sistema de colocación } \\
\text { de recursos, solamente de admi- } \\
\text { nistración). }\end{array}$ \\
\hline Bolsa de valores & $\begin{array}{l}\text { Lugar de transacción de activos } \\
\text { financieros de corto y largo plazo } \\
\text { (Acciones, bonos, y divisas.) }\end{array}$ & $\begin{array}{l}\text { Personas naturales. } \\
\text { Personas jurídicas. }\end{array}$ \\
\hline
\end{tabular}

Fuente: elaboración propia, con del trabajo Acceso a la financiación pymes, de Escobar (2014).

Para demostrar lo expuesto anteriormente, a continuación se presentan, a través de los flujos de caja de financiación, la deuda promedio adquirida por las empresas del sector industrial entre 1995 y 2013 a precios corrientes, recursos que se pueden destinar a diferentes actividades entre ellas inversión.

En el Departamento de Caldas se obtuvieron los siguientes datos: 
Tabla 3. Valor deuda promedio adquirida por las empresas en Caldas durante los años 1995 - 2013 en miles de pesos.

\begin{tabular}{|c|c|}
\hline Departamento / región & $\begin{array}{c}\text { Valor de la deuda promedio adquirida } \\
\text { durante el período 1995 - 2013 en miles } \\
\text { de pesos. }\end{array}$ \\
\hline Caldas & 3.071 .160 \\
\hline
\end{tabular}

Fuente: elaboración propia con datos extraídos de la Superintendencia de sociedades (2014).

En el estudio también se encontró que la correlación entre el crecimiento económico y los flujos de efectivo operativos de las empresas del sector industrial de las regiones se demuestra a partir de dos factores:

Cualitativo. Se establecieron los principales factores que propiciaron el crecimiento económico cada año y para el departamento; estos se relacionaron con el resultado de los flujos de efectivo de la operación, de tal forma que se demuestra la correlación entre las dos variables analizadas (la información fue obtenida de los informes del Banco de la República y el Departamento Nacional de Estadística - DANE):

Gráfica 2. Crecimiento económico y variación de los flujos de efectivo de operación para el departamento de Caldas

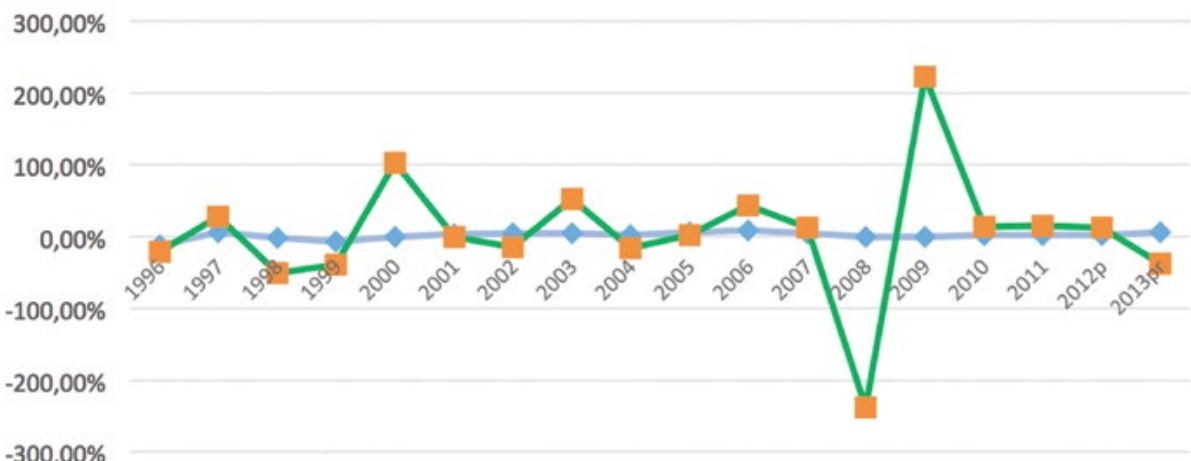

$-300,00 \%$

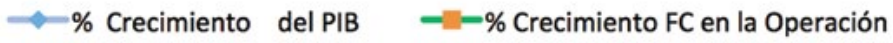

Fuente: elaboración propia con datos del DANE y la Superintendencia de Sociedades.

Se observa en este gráfico la relación entre los hechos económicos que generan el crecimiento económico y los flujos de efectivo; así, de acuerdo con los 
resultados expuestos, se pudo determinar la correlación entre el crecimiento económico y los flujos de efectivo operativos de las empresas en los años 1996, 1997, 1998, 1999, 2000, 2003, 2006, 2010, 2011 y 2012; en la medida en que crecía o decrecía económicamente la región, también lo hacían los flujos de efectivo. Este hecho se confirma con la correlación estadística que se expondrá más adelante. El departamento de Caldas tradicionalmente ha tenido, como principales aportantes al PIB, sectores como el cafetero y el industrial. Sin embargo, en este período se presentó un cambio importante en los sectores que aportan al crecimiento de la economía y los protagonistas son los servicios sociales, los servicios financieros y la construcción (DANE, 2014).

Cuantitativo. A través de una correlación estadística, se establece el grado de correlación ante las variaciones del crecimiento económico y los flujos de efectivo operativo para el conjunto de empresas del departamento. Para establecer esta correlación, se extrajeron los datos del DANE, obteniendo de dicha entidad el PIB por año Caldas (columna b) y luego se calculó la deflactación ${ }^{3}$ a precios de 2008 (columna a) con el propósito de igualar a una misma base tanto el valor del PIB como de los flujos de efectivo. Luego se obtuvo el valor del PIB a precios constantes de 2008 (columna c); para este proceso, se aplicó la siguiente fórmula:

\section{PIB a precios $2008=($ PIB precios corrientes/deflactor $) \times 100$}

Luego, con cada uno de los datos del PIB constantes a precios de 2008, se elaboró el crecimiento económico (columna d), que es el resultado de establecer la variación que presentó el PIB constante durante los años comprendidos entre 1995 y 2013. Para establecer esta variación, se utilizó la siguiente fórmula:

\section{Variación PIB = Ln período actual - Ln del año anterior}

Las fórmulas para deflactar el PIB y el cálculo de su respectiva variación fueron obtenidas del autor William Sharpe (2003).

Finalmente, para establecer la correlación entre las variables, se obtuvieron, de la Superintendencia de Sociedades, los flujos de efectivo operativos para cada una de las empresas en cada año. Luego, se estableció el valor de dichos flujos de efectivo a precios constantes con base en 2008 (columna e), para hacer la comparación de los datos en un mismo momento; así los datos obtenidos por la

3 Consiste en colocar los valores de una serie de tiempo en un mismo periodo o base, con el objeto de analizar los valores de la serie en un periodo de tiempo determinado y poder hacer comparación entre los datos. La base de tiempo la genera el DANE, siendo la última actualización de precios base el año 2008. 
correlación estadística son más homogéneos, lo que permite encontrar conclusiones más confiables.

Tabla 4. Índices de deflactor a precios 2008

\begin{tabular}{|c|c|c|c|c|c|}
\hline Período & $\begin{array}{l}\text { a. Índice De- } \\
\text { flactor }\end{array}$ & $\begin{array}{l}\text { b. Producto } \\
\text { Interno Bruto } \\
\text { para los años } \\
1995-2013 \\
\text { a precios } \\
\text { corrientes }\end{array}$ & $\begin{array}{l}\text { c. Valor del PIB } \\
\text { deflactado a } \\
\text { precios base } \\
\text { año } 2008\end{array}$ & $\begin{array}{l}\text { d. Crecimiento } \\
\text { económico } \\
\text { para Caldas } \\
\text { durante los } \\
\text { períodos } 1996 \\
-2013\end{array}$ & $\begin{array}{l}\text { e. Flujos de } \\
\text { efectivo de- } \\
\text { flactados para } \\
\text { Caldas durante } \\
\text { el período } \\
1995 \text { - } 2013\end{array}$ \\
\hline 1995 & 31,23709 & 1.964 .845 & 6.290 .103 & & \\
\hline 1996 & 37,99651 & 2.112.137 & 5.558 .766 & $-12,36 \%$ & 36.873 .655 \\
\hline 1997 & 44,71589 & 2.655 .671 & 5.938 .987 & $6,62 \%$ & 45.728 .816 \\
\hline 1998 & 52,18481 & 3.057.313 & 5.858 .626 & $-1,36 \%$ & 27.916 .850 \\
\hline 1999 & 57,00236 & 3.125 .523 & 5.483 .147 & $-6,62 \%$ & 20.171 .453 \\
\hline 2000 & 61,98903 & 3.518 .000 & 5.675 .198 & $0,03 \%$ & 56.441 .470 \\
\hline 2001 & 66,72893 & 3.905 .000 & 5.852 .034 & $3,07 \%$ & 54.609 .229 \\
\hline 2002 & 71,39513 & 4.380 .000 & 6.134 .872 & $4,72 \%$ & 44.984 .402 \\
\hline 2003 & 76,02913 & 4.893 .000 & 6.435 .691 & $4,79 \%$ & 72.787 .992 \\
\hline 2004 & 80,20885 & 5.282 .000 & 6.585 .308 & $2,30 \%$ & 60.536 .447 \\
\hline 2005 & 84,10291 & 5.919 .000 & 7.037 .806 & $6,65 \%$ & 57.574 .921 \\
\hline 2006 & 87,86896 & 6.710 .000 & 7.636 .371 & $8,16 \%$ & 81.939 .128 \\
\hline 2007 & 92,87228 & 7.438 .000 & 8.008 .848 & $4,76 \%$ & 88.374 .058 \\
\hline 2008 & 100,00000 & 8.017 .000 & 8.017 .000 & $0,10 \%$ & 8.195 .132 \\
\hline 2009 & 102,00181 & 8.164 .000 & 8.003 .780 & $-0,17 \%$ & 75.903 .238 \\
\hline 2010 & 105,23651 & 8.592 .000 & 8.164 .467 & $1,99 \%$ & 85.617 .721 \\
\hline 2011 & 109,15740 & 9.070 .000 & 8.309.102 & $1,76 \%$ & 97.711 .940 \\
\hline 2012 & 111,81576 & 9.445 .000 & 8.446 .931 & $1,65 \%$ & 108.819.761 \\
\hline 2013 & 113,98254 & 10.172 .000 & 8.924 .174 & $5,50 \%$ & 70.729 .244 \\
\hline
\end{tabular}

Fuente: elaboración propia con datos obtenidos del DANE.

Para establecer la correlación estadística entre el crecimiento económico y los flujos de efectivo de operación en Caldas, se partió del crecimiento económico que presentó entre 1996 y 2013 (véase la tabla 4) y este se relacionó con los flujos de efectivo deflactados que se encuentran en la misma tabla; se obtuvo una correlación de 0,43 , lo cual demuestra que es un resultado bastante bajo para poder realizar predicciones. Con base en esto, se procedió a sumar los flujos 
de efectivo de operación y financiación para determinar la correlación entre las variables. Ello, con el objeto de demostrar que la teoría aplicaba a la realidad en la medida en que al inyectarle liquidez a las empresas, éstas aprovechan estos recursos para hacer inversiones que apuntan a un mayor crecimiento económico

Al realizar esta sumatoria entre los flujos de efectivo de operación y financiación y correlacionarla nuevamente con el crecimiento económico, el resultado de la correlación estadística ascendió al 0,77, con un nivel de significancia de 0,00, lo que demuestra que las variables se pueden utilizar para realizar el análisis de regresión y la posterior predicción de los flujos de efectivo.

En la correlación estadística entre las variables crecimiento económico y flujos de efectivo de operación y financiación, la homogenización de los datos arroja mejores resultados, lo que demuestra que cuando las empresas generan un mayor flujo se podrán dar mejores resultados de crecimiento en la medida que los recursos se destinen al crecimiento (Levine, 1997).

Con la demostración de que los datos son más homogéneos y confiables para realizar la predicción de los flujos, ésta se realiza bajo la siguiente fórmula:

$$
\text { F.E.E. }=\text { Constante }+(C \times \text { C.E. })+\text { Error }
$$

Donde F.E.E. es el flujo de efectivo esperado, C el coeficiente del PIB, C.E. el crecimiento económico esperado. El crecimiento económico esperado se obtiene del Banco Mundial. De acuerdo con las consultas realizadas a varios economistas, los datos que presenta dicha institución son confiables. El error que será utilizado como verificador del modelo para determinar si este es funcional estadísticamente con el indicador de normalidad y homocedasticidad, teniendo en cuenta que el error es para cada una de las observaciones de las variables.

Según las proyecciones de crecimiento del Banco Mundial para el crecimiento de la economía, se obtuvieron los siguientes datos:

Tabla 5. Proyecciones de crecimiento económico para Colombia para los años 2014 - 2017.

\begin{tabular}{|c|c|}
\hline Período & $\begin{array}{c}\text { Crecimiento económico } \\
\text { proyectado }\end{array}$ \\
\hline 2014 & $4,7 \%$ \\
\hline 2015 & $4,4 \%$ \\
\hline 2016 & $4,3 \%$ \\
\hline
\end{tabular}




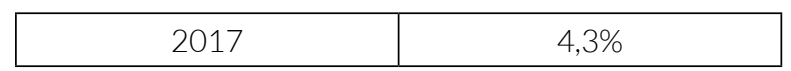

Fuente: elaboración propia con datos extraídos del Banco Mundial (2015).

Se observa en la tabla anterior que las proyecciones del crecimiento económico van disminuyendo año a año, lo que indica que los flujos de efectivo también lo deben hacer de acuerdo con los datos arrojados por la regresión estadística y por la correlación entre el crecimiento económico y los flujos de efectivo. Se establece el crecimiento económico para Colombia puesto que en la búsqueda de la información no hay proyecciones de crecimiento económico para los departamentos o ciudades en particular.

En el proceso de regresión estadística, utilizando las mismas variables y para establecer el modelo adecuado para la predicción de los flujos de efectivo se obtuvieron los siguientes datos:

Tabla 6. Análisis de regresión estadístico para el departamento de Caldas entre los años 1996 - 2013.

\begin{tabular}{|c|c|}
\hline Variables estadísticas & Resultados \\
\hline Constante & 15.798 .824 \\
\hline Coeficiente del PIB & 7.655 .894 \\
\hline
\end{tabular}

Fuente: elaboración propia

Con fundamento el resultado de la tabla anterior para la predicción de los flujos de efectivo futuros se establece la siguiente fórmula:

$$
\text { F.E.E. }=15.798 .824+7.655 .894 \times \text { PIB }
$$

La constante significa que si el valor del PIB de un período es de cero (0\%), es decir, si el crecimiento es nulo, los flujos de efectivo de las empresas del sector industrial en conjunto tendrán un valor de \$15.798.824, el coeficiente del PIB significa que ante una variación del crecimiento en un punto equivalente al $100 \%$, los flujos de efectivo aumentarán en \$7.655.894, también ocurrirá de forma contraria; es decir, si el PIB disminuye en esta cantidad disminuirán los flujos de efectivo futuros. 
Para determinar que el modelo presentado es válido estadísticamente en el departamento de Caldas, se realizaron las siguientes pruebas estadísticas que arrojaron los siguientes datos:

Tabla 7. Pruebas estadísticas del modelo de regresión bivariado para el departamento de Caldas.

\begin{tabular}{|c|c|c|c|c|}
\hline Prueba & $\begin{array}{c}\text { Resultado de la } \\
\text { prueba }\end{array}$ & $\begin{array}{c}\text { Límites de la } \\
\text { prueba }\end{array}$ & $\begin{array}{c}\text { Aceptación de la } \\
\text { prueba }\end{array}$ & $\begin{array}{l}\text { Rechazo de } \\
\text { la prueba }\end{array}$ \\
\hline $\begin{array}{l}\text { Tde Student } \\
\text { Significancia de } \\
\text { la prueba } \\
\text { Coeficiente de la } \\
\text { variable PIB }\end{array}$ & $\begin{array}{c}4,940 \\
0 \% \\
0,77\end{array}$ & $\begin{array}{c}\text { Significancia } \\
<5 \%\end{array}$ & $x$ & \\
\hline $\begin{array}{c}\text { Fisher } \\
\text { Significancia de } \\
\text { la prueba }\end{array}$ & $\begin{array}{c}24,402 \\
0 \%\end{array}$ & $\begin{array}{c}\text { Significancia } \\
<5 \%\end{array}$ & $x$ & \\
\hline $\begin{array}{l}\text { Coeficiente de } \\
\text { determinación }\end{array}$ & 60,4 & $\begin{array}{c}\text { Cerca al } \\
50 \%\end{array}$ & $x$ & \\
\hline $\begin{array}{c}\text { Normalidad } \\
\text { Significancia de } \\
\text { la prueba }\end{array}$ & $\begin{array}{c}0,983 \\
97,4\end{array}$ & $>5 \%$ & $x$ & \\
\hline $\begin{array}{l}\text { Homocedasti- } \\
\text { cidad }\end{array}$ & 30,2 & $>5 \%$ & $x$ & \\
\hline
\end{tabular}

Fuente: elaboración propia con datos extraídos del Banco Mundial (2015).

Según los resultados de la tabla anterior, el modelo es válido para realizar la predicción de los flujos de efectivos futuros ante una variación determinada del $\mathrm{PIB}$, puesto que cumple todas las pruebas estadísticas planteadas.

Para determinar el efecto de la variación económica en los flujos de efectivo, se aplica la fórmula del modelo establecido y se prueba con las variaciones en el 
PIB; para medir el cambio que presentan los flujos de efectivo se ensambló la formula y se realizó una simulación en la que el PIB variaba en un punto porcentual con los siguientes resultados:

Tabla 8. Resultados de la variación de los flujos de efectivo para el departamento de Caldas ante cambios en el PIB en un punto porcentual.

\begin{tabular}{|c|c|c|c|c|c|c|}
\hline Constante & $\begin{array}{c}\text { Coeficiente } \\
\text { del PIB }\end{array}$ & $\begin{array}{c}\text { PIB } \\
\text { ini- } \\
\text { cial }\end{array}$ & $\begin{array}{c}\text { PIB } \\
\text { final }\end{array}$ & $\begin{array}{c}\text { Valor del flu- } \\
\text { jo de efectivo } \\
\text { con el PIB } \\
\text { inicial }\end{array}$ & $\begin{array}{c}\text { Valor del flu- } \\
\text { jo de efectivo } \\
\text { con el PIB } \\
\text { final }\end{array}$ & $\begin{array}{c}\text { Variación } \\
\text { en el } \\
\text { flujo de } \\
\text { efectivo }\end{array}$ \\
\hline 15.798 .824 & 7.655 .894 & $-5 \%$ & $-4 \%$ & 15.416 .029 & 15.492 .588 & $0,494 \%$ \\
\hline 15.798 .824 & 7.655 .894 & $-4 \%$ & $-3 \%$ & 15.492 .588 & 15.569 .147 & $0,492 \%$ \\
\hline 15.798 .824 & 7.655 .894 & $-3 \%$ & $-2 \%$ & 15.569 .147 & 15.645 .706 & $0,489 \%$ \\
\hline 15.798 .824 & 7.655 .894 & $-2 \%$ & $-1 \%$ & 15.645 .706 & 15.722 .265 & $0,487 \%$ \\
\hline 15.798 .824 & 7.655 .894 & $-1 \%$ & $0 \%$ & 15.722 .265 & 15.798 .824 & $0,485 \%$ \\
\hline 15.798 .824 & 7.655 .894 & $0 \%$ & $1 \%$ & 15.798 .824 & 15.875 .383 & $0,482 \%$ \\
\hline 15.798 .824 & 7.655 .894 & $1 \%$ & $2 \%$ & 15.875 .383 & 15.951 .942 & $0,480 \%$ \\
\hline 15.798 .824 & 7.655 .894 & $2 \%$ & $3 \%$ & 15.951 .942 & 16.028 .501 & $0,478 \%$ \\
\hline 15.798 .824 & 7.655 .894 & $3 \%$ & $4 \%$ & 16.028 .501 & 16.105 .060 & $0,475 \%$ \\
\hline 15.798 .824 & 7.655 .894 & $4 \%$ & $5 \%$ & 16.105 .060 & 16.181 .619 & $0,473 \%$ \\
\hline 15.798 .824 & 7.655 .894 & $5 \%$ & $6 \%$ & 16.181 .619 & 16.258 .178 & $0,471 \%$ \\
\hline
\end{tabular}

Fuente: elaboración propia.

De acuerdo con esta tabla, se comprueba en este modelo que siempre que el crecimiento económico sea cero por ciento (0\%), el valor de los flujos de efectivo será igual al valor de la constante y por cada punto porcentual que varíe el PIB, los flujos de efectivo variarán en $0,48 \%$ en promedio. Las variaciones en el PIB se realizaron desde $-5 \%$ hasta $6 \%$, ya en esta escala de variación PIB se distribuyen normalmente los datos.

Según esto, si se desea establecer la variación que tendrán los flujos de efectivo del sector industrial en el departamento de Caldas, se debe utilizar esta variación obtenida; es decir, si el PIB esperado aumenta en un punto porcentual debe incrementar sus flujos de efectivo en $0,48 \%$ para el próximo período y si es un decremento en este porcentaje, debe reducir su flujo en $-0,48 \%$. También por cada variación en $0,1 \%$ o $-0,1 \%$ debe incrementar o reducir su flujo en $0,048 \%$ y $-0,048 \%$ respectivamente. 
Las variaciones en el PIB esperadas según los datos del Banco Mundial serán en 2014 del 4,7\% y en 2015 del 4,4\%. Por tanto, la variación del PIB será de $-0,3 \%$, lo que significa que los flujos de efectivo deben variar en $-0,14 \%(-0,048 \%$ x 3 x 100). Y en 2016 el crecimiento en el PIB esperado es del 4,3\%, pues la variación con respecto al año 2015 es de $-0,1 \%$, por lo cual los flujos de efectivo deben disminuir en $-0,048 \%$ (-0,048\% x 1 x 100). En 2017 no se espera variación en el PIB porque no se generará variación en los flujos de efectivo. Lo anterior como se presenta en la siguiente tabla:

Tabla 9. Predicción de los flujos de efectivo para los años 2014 - 2017 para las empresas del sector industrial del departamento de Caldas.

\begin{tabular}{|c|c|c|c|}
\hline Período & PIB proyectado & $\begin{array}{c}\text { Valor esperado } \\
\text { de los flujos de } \\
\text { efectivo }\end{array}$ & $\begin{array}{c}\text { Variación de } \\
\text { los flujos de } \\
\text { efectivo }\end{array}$ \\
\hline 2014 & $4,7 \%$ & 16.158 .651 & \\
\hline 2015 & $4,4 \%$ & 16.135 .683 & $-0,142 \%$ \\
\hline 2016 & $4,3 \%$ & 16.128 .027 & $-0,047 \%$ \\
\hline 2017 & $4,3 \%$ & 16.128 .027 & $0,000 \%$ \\
\hline
\end{tabular}

Fuente: elaboración propia.

Las conclusiones se enmarcan de acuerdo con los objetivos planteados, donde inicialmente se muestra el uso de variables adicionales para facilitar el proceso de predicción de los flujos de efectivo; seguidamente se define como la liquidez es la variable fundamental que relaciona las variables analizadas y finalmente se ilustra sobre la relación estadística entre las variables, las cuales permite realizar las futuras predicciones.

Sloan (1996), Maya (2002) y Gabás (1994), señalan que la predicción de los flujos futuros de efectivo de las empresas se debe realizar a través de variables internas tales como ingresos históricos, capital de trabajo, precios históricos de las acciones, inventarios, etc. Sin embargo, Ismail y Choi (1996), trataron de explicar los futuros flujos de efectivo a través de la relación que podrían tener estos con algunos factores económicos como la competencia, el tamaño de la empresa y el tipo de producto que esta ofrece. Pero ninguno de ellos logró hacer predicciones y encontraron que algunas variables se correlacionan mejor que otras con los flujos de efectivo. 
En este contexto, lo propuesto en el presente trabajo no quiere determinar que los usos de otras variables no son importantes en la predicción de los flujos de efectivo, pero es preciso tener en cuenta otras como el crecimiento de la economía, donde se encuentra el vacío teórico que se trabajó en este análisis.

La relación entre el crecimiento económico y los flujos de efectivo es la liquidez con la cual cuentan las empresas para destinar recursos a la inversión, que apoyará posteriormente el crecimiento económico. Sin embargo, las empresas no tienen en muchos casos, los recursos o la liquidez suficiente para llevar a cabo dichas inversiones. Por tanto, recurren al sistema financiero para recoger los recursos adicionales que puedan apalancar la inversión (Levine, 1997). Por eso, se encontró evidencia empírica respecto a que casi el 100\% de las empresas analizadas, recurrieron al crédito financiero para apalancar sus operaciones y sus inversiones.

En el departamento de Caldas, el 56\% de los años que se analizaron presenta correlación directa entre las variables crecimiento económico y flujos de efectivo. Esta región se caracteriza porque el sector cafetero y el sector industrial han sido los protagonistas para la generación del crecimiento de la economía regional. En los últimos años, sin embargo, estos han pasado a un segundo renglón y sectores como la construcción y los servicios están apoyando al crecimiento del PIB. Conviene destacar que las industrias del departamento en sus flujos de efectivo dependen de los resultados de las exportaciones y los ingresos que se obtienen a nivel nacional.

Se concluye que en la medida que los flujos de efectivo reciban mayor liquidez, esta disponibilidad de recursos adicionales mejora la correlación entre las variables. En el desarrollo del presente trabajo, se realizó una comparación de correlación entre el crecimiento económico y los flujos de efectivo de operación del sector industrial.

Además, se realizó la correlación entre el crecimiento económico y la sumatoria entre los flujos de efectivo de operación y financiación, llegando al 0.77, lo que significa que al inyectarle liquidez a las empresas, éstas tienen mayor disponibilidad de recursos que se destinan a la inversión que deriva en crecimiento económico, como lo indica Levine (1997). Esta mayor liquidez la proporcionan 
los flujos de efectivo de la financiación, que son el resultado de obtener recursos monetarios a través del sistema financiero (Terceño y Guercio, 2011).

\section{Referencias}

Aceves, S. y Martínez, J. (2013). The financial system and his impact in the private sector dynamics. Revista Contaduría y administración, 58, 175 - 199.

Aranaz, M. (2002). SPSS para Windows. Madrid, España: Mc Graw Hill interamericana.

Banco Mundial. (2015). Real GDP growth at markets prices in percent and current account balance in percent of GDP, unless indicated otherwise. Recuperado de: www.worldbank.org/content/dam/worldbank/GEP/GEP2015a/pdfs/ GEP2015a_chapter2_regionaloutlook_LAC.pdf

Banrepública (2012). Informe de coyuntura económica regional. Recuperado de: http://www.banrep.gov.co/sites/default/files/publicaciones/archivos/ icer_caldas_2012.pdf

Creswell, J. (2005). Educational research: planning, conducting and evaluating quantitative and qualitative research. Upper saddle river. Pearson education.

Departamento Administrativo Nacional de Estadística, DANE. (2014). Estadísticas, Producto Interno Bruto. Recuperado de: www.dane.gov.co/index. $\mathrm{php} / \mathrm{esp} / \mathrm{pib}-\mathrm{cuentas-nacionales/investigaciones-especiales/77-cuen-}$ tas-nacionales/cuentas-anuales/

Escobar, G. (2014). Acceso a financiación de las pymes del sector comercio para la ciudad de Manizales. Manizales, Colombia: Universidad Autónoma de Manizales.

Fisher, R. (1925). Statistical Methods for Research Workers. Oxford, Inglaterrra: Clarendon Press.

Gabás, F. (1994). Capacidad predictiva de los componentes del beneficio: flujos de tesorería y ajustes corto - largo plazo. Revista española de financiación y contabilidad, 24(78), $107-142$. 
Ismail, B. y Choi, K. (1996). Determinats of time - series propierties of earnings and cash flows. Review of financial economics, 5, $131-145$.

León, O. y Montero, I. (2003). Métodos de Investigación en psicología y Educación. Madrid, España: McGraw-Hill.

Levine, R. (1997). Desarrollo Financiero y Crecimiento económico: Enfoques y temario. Journal of economic literature, 35, $688-726$.

Maya, C. (2002). Evidencia empírica sobre la utilidad de la información financiera para la predicción de los resultados futuros. Revista española de financiación y contabilidad, 31(111), $189-224$.

Ruiz, A. (2004). Mercados financieros y crecimiento económico en América Latina: un análisis econométrico. Revista análisis económico, vol, XIX, 141- 165.

Sampieri, R., Collado, C. y Lucio, P. (2006). Metodología de la Investigación. México DF: Mc Graw Hill.

Sloan, R. G. (1996). Do stock process fully reflect information in accruals and cash flows about future earnings? The accounting review, $71(3), 289-315$.

Solow, R. (1956). A contribution to the theory of economic growth. Quarterly Journal of Economics, 78, 65-94.

Supersociedades. (2014). Sirem, estados financieros sociedades año 1995 - 2012. Recuperado de: www.supersociedades.gov.co

Terceño, A. y Guercio, M. (2011). Economic growth and development of the financial system. A comparative analysis. investigaciones Europeas de dirección y economía de la empresa, 17, $33-46$. 